Z Rheumatol 2006 $65: 182-182$ DOI 10.1007/s00393-006-0047-2

Online publiziert: 28. Februar 2006

๑) Springer Medizin Verlag 2006

\author{
W. Gross ${ }^{1} \cdot$ E. Märker-Hermann ${ }^{2}$ \\ ${ }^{1}$ Poliklinik für Rheumatologie, Universitätsklinikum Schleswig- \\ Holstein, Campus Lübeck, und Rheumaklinik Bad Bramstedt \\ ${ }^{2}$ Dr. Horst Schmidt-Kliniken, Klinikum der Landeshauptstadt Wiesbaden, Wiesbaden
}

\title{
Prof. Dr. W. Keitel wird 75!
}

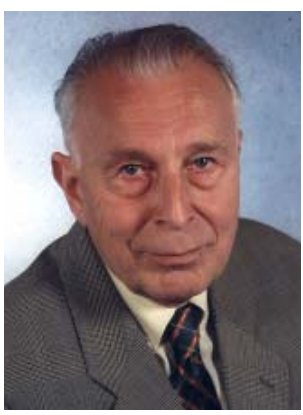

Dervon uns allen sehr geschätzte Rubrikenherausgeber dieser Zeitschrift - er ist seit 1997 Ehrenmitglied der Deutschen Gesellschaft für Rheumatologie - wird am 2. April einen besonderen Geburtstag feiern: Prof. Dr. med. Wolfgang Keitel, geb. am 2.04.1931 in Neumark, Kreis Merseburg, wird 75 Jahre alt! $\mathrm{Zu}$ diesem besonderen Ehrentag möchten wir sowohl für die Herausgeber als auch den Vorstand und Beirat der DGRh dem Jubilar herzlich gratulieren.

Prof. Keitel hat nach seinem Medizinstudium an der Martin-Luther-Universität Halle seine internistische Ausbildung an der Medizinischen Klinik der Medizinischen Akademie Magdeburg erhalten. Sicherlich war die dann auch dort durchgeführte Doktorarbeit über Komplementbestimmungstechniken für die weitere berufliche Entwicklung mit ausschlaggebend. Auf jeden Fall hat er sich schon früh auch in der allgemein-internistisch ausgerichteten Universitätsklinik für Rheumatologie und Immunologie interessiert, diese Kombination auch als Oberarzt im Klinikum vertreten und sich schließlich dann mit dem Thema "Gefäßantikörper" nach tierexperimentellen und klinischen Arbeiten habilitiert. Im Weiteren hat Wolfgang Keitel dann die Rheumatologische Klinik im Bezirkskrankenhaus Magdeburg in Vogelsang/Gommern aufgebaut und als Chefarzt geleitet.

Seine hervorragenden wissenschaftlichen und klinischen Leistungen führ- ten dazu, dass er sowohl Vorstandsmitglied der Gesellschaft für Rheumatologie der DDR als auch Vertreter seiner Gesellschaft im ständigen Komitee „Internationale Klinische Studien“ der EULAR wurde. In den Jahren 1974-76 war er Vorsitzender dieser Gesellschaft und hat in den späteren Jahren die Ehrenmitgliedschaft der rheumatologischen Gesellschaften der Tschechoslowakei (1984), Polens (1987) und dann 1997 unserer DGRh erhalten. 1990 wurde Prof. Keitel in den Beirat der DGRh gewählt und war nahezu zehn Jahre lang Sprecher der Kommission „Qualitätssicherung" der DGRh.

Seine Publikationen, und zwar wissenschaftliche sowie Fort- und Weiterbildungsarbeiten, gehen weit über die Zahl von 300 hinaus. Darunter finden sich u.a. sein „Bewegungsfunktionstest", der auch seinen Weg in das internationale Schrifttum und damit in Handbücher der Rheumatologie gefunden hat. Unserer Leserschaft ist Prof. Keitel vor allen Dingen über seine medizingeschichtlichen Darstellungen bekannt, die er uns unermüdlich und ohne Aufforderung kontinuierlich Heft für Heft zur Verfügung stellt. Hierfür und natürlich auch für die nicht genannten Leistungen für die gesamte Rheumatologie möchten wir uns bedanken und dem Jubilar mit seinen Angehörigen nicht nur gratulieren, sondern auch weiterhin dieselbe Schaffenskraft für die Zukunft wünschen: ad multos annos Wolfgang Keitel!

Wolfgang L. Gross

Elisabeth Märker-Hermann 